\title{
A Survey on Routing Protocols in Wireless Multimedia Sensor Networks
}

\author{
Fernaz Narin Nur \\ Lecturer \\ Daffodil International University
}

\author{
Nazmun Nessa Moon \\ Senior Lecturer \\ Daffodil International University
}

\author{
Narayan Ranjan \\ Chakraborty \\ Lecturer \\ Daffodil International University
}

\begin{abstract}
The recent technological advances in micro electromechanical systems have promoted the development of a powerful class of sensor-based distributed intelligent systems capable of ubiquitously retrieving multimedia information, namely Wireless Multimedia Sensor Networks (WMSNs). WMSNs are gaining more popularity day by day as they are envisioned to support a large number of both non-real time and real-time multimedia applications. In this paper, current state-of-the-art in energy-efficient routing techniques for WM-SNs is surveyed together with the highlights of the performance issues of each strategy. This paper outlines the design challenges of routing protocols for WMSNs. Architectures for WMSNs are explored, along with their advantages and drawbacks. Further, a classification of recent routing protocols for WMSNs and a discussion of possible future research trends are presented.
\end{abstract}

\section{General Terms}

Your general terms must be any term which can be used for general classification of the submitted material such as Pattern Recognition, Security, Algorithms et. al.

\section{Keywords}

Wireless Multimedia Sensor Networks (WMSNs), Micro Electro-Mechanical systems (MEMS), Quality-of-Service (QoS).

\section{INTRODUCTION}

The major objectives behind the research and exploitation of Wireless Multimedia Sensor Networks (WMSNs) lie in two broad aspects: event detection and data communication through node coordination. On the other hand, today's wireless communication is gradually changing the paradigms from the existing scalar services like light, temperature, etc. to a new world of real-time audio-visual applications. The increasing popularity of multimedia applications has already given birth to the new term Wireless Multimedia Sensor Networks (WMSNs). Wireless Multimedia Sensor Networks are a class of Wireless Sensor Networks where the carried flows are mainly composed of images and/or video streams. With the availability of low cost CMOS cameras and microphones coupled with the significant progress in distributed signal processing and multimedia source coding techniques, WMSN are forecast to become highly integrated into our daily activities with applications spanning over a large panel of domains and contexts.

The main characteristics of a WMSN include: Power consumption constrains for nodes using batteries or energy harvesting, Ability to cope with node failures, Mobility of nodes, Communication failures, Heterogeneity of nodes,
Scalability to large scalability to large scale of deployment, Ability to withstand harsh environmental conditions. Most deployed WSNs measure physical phenomena like temperature, pressure, humidity, or location of objects. In general, most of those applications have low bandwidth demands, and are usually delay tolerant [1]. On the other hand, WMSNs, comprised of sensor devices equipped with audio and visual information collection modules, can have the ability to retrieve multimedia data, store or process data in real-time, correlate and fuse multimedia data originated from heterogeneous sources, and wirelessly transmit collected data to desired destinations [1].

WMSNs have not only stretched the horizon of traditional sensor networks, but also proliferated significantly to a multiplicity of novel applications. Recent years have witnessed the pilot deployments of WMSNs for a class of real-time mission critical and monitoring applications, including search and rescue, security surveillance, traffic and environmental monitoring, wild animal tracking, disaster management, and patient monitoring. For example, WMSNs have recently acquired particular interest for their possible use to monitor the elderly aging at home [2]. In fact, processing video with sensor networks eliminate the need for elderly to remember to wear cumbersome instrumentation and sensors. A sensor network for health-care environment uses video transmission as sensory modality for identifying patients' behavior [2]. Another example is multimedia surveillance systems, where many different media streams (audio, video, images, textual data, sensor signals, etc.) are concerned to provide an automatic analysis of controlled environment and real-time interpretation of the scene. Video and audio sensors are utilized as multimedia facilities to enhance and complement existing surveillance systems against crime and terrorist attacks. Dependable and large scale video and audio sensor networks, to some extent, extend the ability of law enforcement agencies to monitor areas, public events, private properties and borders [1]. Moreover in some monitoring and security applications, non-real time audio-visual information is also beneficial to infer and record potentially relevant activities (thefts, car accidents, traffic violations) to make reports available for future query [1]. There are several other fields where WMSNs have presented unprecedented potential for applications requiring ubiquitous access to both real-time and non real-time data.

The remainder of the paper is organized as follows:

The most considerable factors for WMSNs are discussed in section 2. In Section3 classification of network architecture according to various applications is discussed. Then, in section 4 existing routing approaches for traditional sensor networks are discussed along with their limitations to work 
for WMSNs. In section 5 some open issues and research directions are identified with concluding remarks are given.

\section{CONSIDERABLE FACTORS FOR WMSNS}

Wireless Multimedia Sensor Networks (WMSNs) will be enabled by the convergence of communication and computation with signal processing and several branches of control theory and embedded computing [1]. This section presents the most dominant and challenging factors that need be addressed in order to achieve effective communication in WMSNs. Multimedia applications generate high volumes of traffic, which not only require high transmission rates, but also extensive processing. Energy-aware routing protocols for WMSNs should be designed while accounting for all forms of energy consumption. Power consumption is also a fundamental concern in WMSNs, even more than in traditional wireless sensor networks. Therefore, protocols, algorithms and architectures to maximize the network lifetime while providing the QoS required by the application are a critical issue.

The wide variety of applications envisaged on WMSNs will have different requirements. In addition to data delivery modes typical of scalar sensor networks, multimedia data include snapshot and streaming multimedia content. [3] [4]. Snapshot type multimedia data contain event triggered observations obtained in a short time period. Streaming multimedia content is generated over longer time periods and requires sustained information delivery. Hence, a strong foundation is needed in terms of hardware and supporting high-level algorithms to deliver QoS and consider applicationspecific requirements. Uncompressed raw video streams require excessive bandwidth for a multi-hop wireless environment. Hence, it is apparent that efficient processing techniques for lossy compression are compulsory for multimedia sensor networks. Predictive encoding requires complex encoders, powerful processing algorithms, and entails high energy consumption. However, it has recently been shown [5] that the traditional balance of complex encoder and simple decoder can be reversed within the framework of the so-called distributed source coding, which exploits the source statistics at the decoder, and by shifting the complexity at this end, allows the use of simple encoders. Clearly, such algorithms shows potential for WMSNs and especially for networks of video sensors, where it may not be reasonable to use existing video encoders at the source node due to processing and energy constraints. It is of fundamental importance for the commercial development of sensor networks to provide services that allow querying the network to retrieve useful information from anywhere and at any time. For this reason, future WMSNs will be remotely accessible from the Internet, and will therefore need to be integrated with the IP architecture.

\section{NETWORK ARCHITECTURE FOR WMSNs}

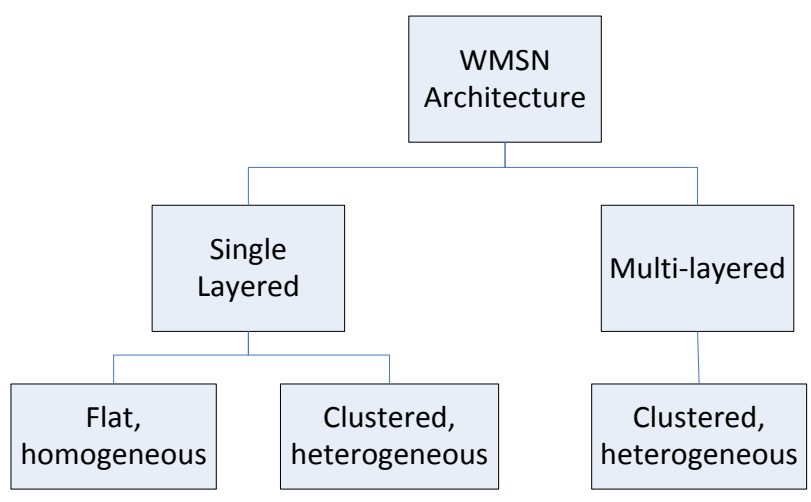

Fig. 1: Classification of the architecture of WMSNS.

Based on the necessities of different applications, the architecture of WMSNs can be alienated into three different classes: single layered flat and homogeneous; single layered clustered and heterogeneous; and multi-layered clustered and heterogeneous as in fig.1. In single layered flat and homogeneous architecture, each multimedia sensor node has the same physical capabilities, and a subset of the deployed sensors has higher processing capabilities termed as multimedia processing hubs, which are responsible for innetwork processing (i.e., data aggregation, discard of redundant data) in a distributed fashion and relaying to a sink through a multi-hop path [1] [6] [7]. Single layered clustered and heterogeneous architecture is composed of heterogeneous sensory nodes (multimedia sensor nodes, basic WSN or scalar sensor nodes, etc.), where the sensor nodes in the cluster gather sensory information from the environment and then send it to the cluster head, which has more resources and computational power as compared to other cluster nodes. The processed information is then transmitted to the sink via wireless link by the cluster head. The multilayered clustered and heterogeneous architecture is comprised with a layer of scalar sensor nodes for performing simple tasks of gathering the scalar information from the surrounding ambience, a layer of medium resolution multimedia sensor nodes capable of gathering multimedia information from the surrounding environment, and a final layer of highly powerful multimedia sensor nodes for performing complex tasks and transmitting data to the sink.

Most proposals for wireless sensor networks are based on a flat, homogenous architecture in which all sensors has the same physical capabilities and can only interact with neighboring sensors. Traditionally, the research on algorithms and protocols for sensor networks has focused on scalability, i.e., how to design solutions whose applicability would not be limited by the growing size of the network. Flat topologies may not always be suited to handle the amount of traffic generated by multimedia applications including audio and video. Likewise, the processing power required for data processing and communications, and the power required to operate it, may not be available on each node. 


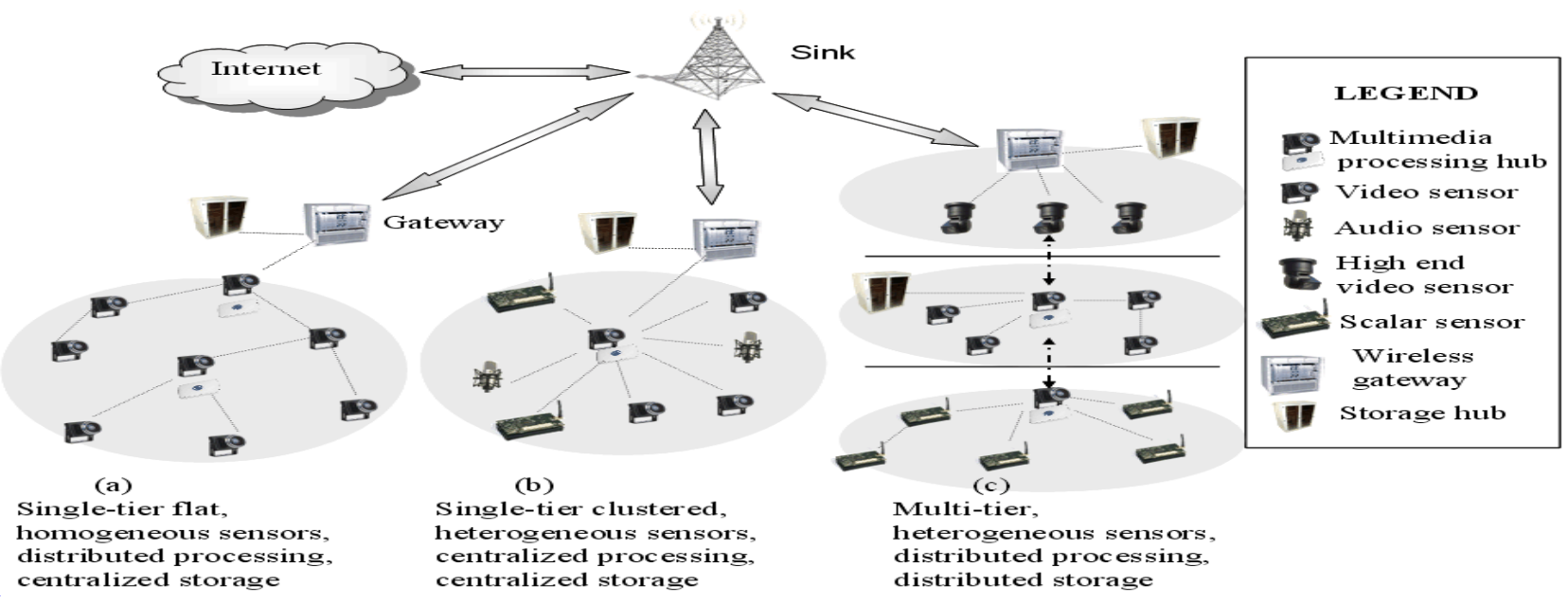

Fig. 2 Reference architecture of a wireless multimedia sensor network

The reference architecture for WMSNs has introduced in fig.2 [1]. There are three sensor networks with different characteristics deployed in different physical locations. The first cloud on the left shows a single-tier network of homogeneous video sensors where subsets of the deployed sensors have higher processing capabilities, and are thus referred to as processing hubs. The union of the processing hubs constitutes a distributed processing architecture. The multimedia content gathered is relayed to a wireless gateway through a multi-hop path. The gateway is interconnected to a storage hub that is in charge of storing multimedia content locally for subsequent retrieval. Clearly, more complex architectures for distributed storage can be implemented when allowed by the environment and the application needs, which may result in energy savings since by storing it locally, the multimedia content does not need to be wirelessly relayed to remote locations. The wireless gateway is also connected to a central sink, which implements the software front-end for network querying and tasking. The second cloud represents a single-tiered clustered architecture of heterogeneous sensors (only one cluster is depicted). Video, audio, and scalar sensors relay data to a central cluster head, which is also in charge of performing intensive multimedia processing on the data (processing hub). The cluster head relays the gathered content to the wireless gateway and to the storage hub. The last cloud on the right represents a multi-tiered network, with heterogeneous sensors. Each tier is in charge of a subset of the functionalities. Resource-constrained, low-power scalar sensors are in charge of performing simpler tasks, such as detecting scalar physical measurements, while resource-rich, high-power devices are responsible for more complex tasks. Data processing and storage can be performed in a distributed fashion at each different tier.

\section{ROUTING PROTOCOLS FOR WMSNs}

Many energy-aware routing protocols have been proposed and evaluated for WMSNs in the past few years, and in this section, the energy-efficient routing approaches are. According to the current research trend, the routing protocols are classified mainly according to the type and number of QoS constraints they consider. Again, we describe the routing approaches based on type of data they handle (still vs. streaming data), type of data delivery model they use (query driven, even driven), classes of algorithms (genetic algorithm, supervised learning, clustered-control algorithm etc.) they adopt, and the hole-bypassing approaches they use.

Data collected by the sensor nodes needs to be sent to the sink, where useful information can be extracted from it. Comprehensive surveys of the major routing schemes existing in the literature are presented in [8] [6]. The concerns of routing in general differ significantly from the specialized service requirements of multimedia streaming applications. As an example, multiple routes may be necessary to satisfy the desired data rate at the destination node. Also, different paths exhibiting varying channel conditions may be preferred depending on the type of traffic and its resilience to packet loss. Next the various approaches to routing in WMSNs is discussed the various approaches to routing in WMSNs while maintaining that protocols may incorporate features from more than one of the following classes.

\subsection{Protocols providing real time delay guarantee}

In the recent research work, some routing protocols are reviewed depending on real time delay guarantee; this real time delay can be either soft or hard. Sequential Assignment Routing (SAR) [9] is the first routing protocol considering QoS (e.g., end-to-end delay) and energy efficiency for sensor networks which includes a table driven multi-path routing and path restoration technique to create trees routed from one hop neighbor of the sink. The objective of the SAR routing algorithm is to minimize the average weighted QoS metric throughout the lifetime of the network. The multipath routing scheme ensures fault-tolerance, and path restoration technique eases the recovery in case of node failure. The limitation of SAR approach is that it suffers from the overhead of maintaining the tables and status information for each sensor node when number of nodes is huge.

RAP [4] is a soft real time delay bounded location aware and priority-based routing protocol which pioneers in considering deadline issues in multi-hop wireless multimedia sensor networks. RAP provides convenient mechanisms to be used in both query-initiated and event-initiated applications. RAP implements a differentiated priority-based Velocity Monotonic Scheduling (VMS) policy, which is based on a notion of packet requested velocity suitable for packet scheduling in sensor networks. Each packet is expected to make its end to end deadline if it can move toward the 
destination at its requested velocity, which reflects its local urgency. Compared with non-prioritized packet scheduling, VMS improves the deadline miss ratios of sensor networks by giving higher priority to packets with higher requested velocities. Also, VMS can perform better than deadline based packet scheduling because velocity reflects the local urgency at each hop more accurately when packets with the same deadline have different distances to their destinations. Since the requested velocity can be locally determined assuming that each sensor node knows its own location, a combination of VMS and geographic forwarding (GF) provides a localized and scalable protocol for real-time multimedia communication on sensor networks. But for multimedia data, such greedy forwarding can deplete the bandwidth quickly. The limitation of RAP is that it does not consider any alternate approach for such problem. Again, while calculating priority, the scheme does not consider the number of hops that the packet has to traverse in deciding the priority. Most importantly, there is no direct metric to show how energy is conserved in multimedia routing.

SPEED [3] is proposed to support real time traffic with delay requirements while considering prioritization. SPEED is a spatio-temporal, priority-based, QoS aware routing protocol for sensor networks that provides soft real-time, end-to-end delay guarantees. The protocol requires that each node maintain localized information with minimal control overhead, and uses nondeterministic geographic forwarding to find paths. The main objective of this work is to support a spatio-temporal communication service with a given maximum delivery speed across the network. The routing module in SPEED is called Stateless Geographic Non Deterministic forwarding (SNFG), and works with other four modules at the network layer Real-time Power Aware Routing (RPAR) [7] protocol pioneers the approach of incorporating energy efficiency in real-time communication. RPAR achieves application specific end to end delay guarantee at low power by dynamically adjusting transmission power and routing decisions based on the workload and packet deadlines. Another distinctive feature of RPAR is that it calculates average link quality taking link variability into consideration. RPAR also handles realistic and dynamic properties of WMSNs such as lossy links, limited memory, and bandwidth. The usage of localized information adds scalability feature with RPAR. In addition, the novel neighborhood management mechanism apart from periodic beaconing scheme adopted by SPEED [3] and MMSPEED [10] is used to maintain and discover neighboring nodes. The unique forwarding policy and neighborhood management of RPAR together can introduce significant power reduction hence results in prolonged network life time with desired real-time guarantee. The drawback of the protocol is that it shows degraded performance in handling large hole and sudden congestion. Ergen et al. [11] propose a power efficient routing algorithm that ensures hard delay guarantee for WMSNs. It adopts a single sink model and aims at maximizing the lifetime of a WMSN by adjusting the number of packets traversing throughout the network. To achieve this goal, first the delay constraint is excluded and lifetime maximization is formulated as a linear programming (LP) problem, and solution is implemented in a distributed manner which uses an iterative algorithm to approximate the centralized optimal one. Then, delay guarantee is ensured into the energy efficient routing by limiting the length of routing paths from each node to the sink. The protocol can guarantee end-to-end delay requirement and prolong network lifetime. However, it is noticeable that it is not flexible enough to meet application specified delay bound generally.

\subsection{Routing for video streaming}

In WMSNs, real time video streaming data generally has a soft deadline, calling then for shortest path routing approaches with the minimum end-to-end delay. Also, transmission requirements in terms of bandwidth can be several times higher than the maximum transmission capacity (bandwidth) of sensor nodes, needing then multipath routing. Some protocols are proposed to explicitly handle real time streaming by taking both end-to-end latency and bandwidth into consideration.

Optimized energy-delay sub-network routing (OEDSR) [12] is a cluster-based event-driven multi hop energy efficient approach addressing the end-to-end delay constraint. The fully distributed OEDSR protocol calculates the available energy, average end-to-end latency values of the links and the distance from the sink to determine the best next-hop forwarding node. The protocol ensures that the selected path from the cluster head to sink to be loop-free, power-efficient and has the least end-to-end delay. In addition, the lifetime of the network is maximized since the energy is taken into account while selecting nodes from a route. Moreover, when a node loses more energy an alternate path is computed to maintain the load of the network. OEDSR achieves lower average end-to-end delay, fewer collisions and less energy consumption than DSR [13].

Directional Geographical Routing (DGR) [14] protocol, which investigates H.26L real-time video communications in WMSNs, where video streams are transmitted under a number of resource and performance constraints, such as bandwidth, energy and delay. DGR divides a single video stream into multiple sub-streams and exploits multiple disjoint paths to transmit these sub-streams in parallel in order to make the best of limited bandwidth and energy in WSNs and to achieve a reliable delivery. Simulation results show that DGR exhibits high delivery ratio and low end-to-end delay. However, it tackles path failures with local repairs at the cost of additional overheads and transmission latency. In addition, DGR assumes that any node can send video packets to the sink at any instance, which limits its practicality of deploying it for large networks.

\subsection{Swarm intelligence based routing}

Ant-based Service Aware Routing (ASAR) [15] is a hierarchical protocol that incorporates reinforcement learning to routing. ASAR periodically chooses three suitable paths to meet diverse QoS requirements from different kind of services (event-driven, data query and stream query) by positive feedback mechanism used in ant-based algorithms, thus maximizing network utilization and improving network performance. It maintains optimal path table and pheromone path table at each cluster head. Routing selection for different data services is made based on delay, packet loss rate, bandwidth and energy consumption required by the type of traffic. Besides the bottleneck problem of hierarchical models, new optimal path setup due to congestion requires extra calculation which may decrease network performance by engaging extra energy for large networks.

Peng et al. [16] propose an adaptive QoS and energy aware routing approach that uses an improved biological ant colony algorithm for WMSNs not only to meet QoS requirements in 
an energy-aware fashion, but also balance the node energy utilization to maximize the network lifetime. The special forwarding ants are used to discover optimum routing paths between the sensor nodes and the sink nodes in terms of distance, delay, packet loss, bandwidth, and energy levels. Extensive performance analysis under different network load is yet to be performed.

\subsection{Clustered control based routing}

Haiping and Ruchuan [13] propose a novel clustered control algorithm based on location information, energy, priority of coverage and multi-layered architecture, which is different from connection prediction scheme [25] and two-hop clustered image transmission scheme [17]. This approach selects a cluster-head according to geographical locations and remained energy at the nodes and ensures the higher coverage rate for the cluster head by a priority mechanism to avoid the concentrated and marginal distribution of cluster-heads. This approach reduces the energy cost by increasing the sleeping nodes during non-media data transmission phase and adding many intermediate nodes to forward data during multimedia media data transmission which in turn prolongs the lifetime of the network. Simulation results show that this protocol achieves prolonged service time and decreased energy consumption than GAF [18] and ASCENT [19]. The limitation of this approach is lack of showing experimental results in claiming that it improves reliability, load-balancing, uniform distribution of cluster-heads and strengthens the backbone routing paths in the networks.

\section{CONCLUSION AND FUTURE WORK}

There is no doubt that energy efficiency should get the foremost importance in designing routing protocols for sensor networks. But for WMSNs, the QoS requirements must have equal importance. So, there is always a trade-off between multimedia service guarantee and energy efficiency. There is still a lack of practical solutions. The net benefits and the practicality of these techniques still need to be demonstrated. It is necessary to fully explore the trade-offs between the achieved fidelity in the description of the phenomenon observed, and the resulting energy consumption. As discussed above, there is a need for high layer abstractions that will allow fast development of sensor applications. However, due to the resource-constrained nature of sensor systems, it is necessary to control the efficiency of the low-level operations performed on battery limited and resource-constrained sensor nodes. Routing in sensor networks maintains neighbor information on neighbors' states and potentially many other factors in order to make informed decisions. Challenges arise in performing accurate and adaptive information discovery.

For routing in WMSNs, supervised and reinforcement learning based algorithms are interesting classes of algorithms that require further explorations. Current routing protocols mainly focus on optimizing application specific nature of the sensor networks, but do not take security into account. But WMSNs transmit multimedia information which provides more detailed information. Such information leakage can be more malicious. In this regard, secure routing is an issue that needs further attention.

The common objective of research based on WMSNs is to provide QoS guarantee while using energy conserving strategies. This paper present a comprehensive review on research challenges and the state-of-the art of energy-aware routing techniques for WMSNs, and highlight the advantages and performance issues of each routing protocol and algorithm. Finally, open issues are provided in order to stimulate more research interests in those unexplored areas.

\section{REFERENCES}

[1] Ian F. Akyildiz, Tommaso Melodia, and Kaushik R.Chowdhury. A survey on wireless multimedia sensor networks. Comput. Netw., 51(4):921-960, March 2007.

[2] Thiago Teixeira, Eugenio Culurciello, Joon HyukPark, Dimitrios Lymberopoulos, Andrew Barton-Sweeney, and Andreas Savvides. Address-event imagers for sensor networks: evaluation and modeling. In Proceedings of the 5th international conference on Information processing in sensor networks, IPSN '06, pages 458-466, New York, NY,USA, 2006. ACM.

[3] Tian He, John A Stankovic, Chenyang Lu, and Tarek Abdelzaher. Speed: A stateless protocol for real-time communication in sensor networks.2003.

[4] Chenyang Lu, Brian M. Blum, Tarek F. Abdelzaher, John A. Stankovic, and Tian He. Rap: A real time communication architecture for large-scale wireless sensor networks. 2002.

[5] Lucian Ciobanu and Luis Corte-Real. Multi view codec with low-complexity encoding for distributed video coding. Multimedia Tools and Applications, January 2012.

[6] Jamal N. Al-karaki and Ahmed E. Kamal. Routingtechniques in wireless sensor networks: A survey. IEEE Wireless Communications, 11:6-28,2004.

[7] Yinsheng $\mathrm{Xu}$, Fengyuan Ren, Tao He, Chuang Lin, Canfeng Chen, and Sajal K. Das. Real-time routing in wireless sensor networks: A potential field approach. ACM Trans. Sen. Netw., 9(3):35:1-35:24, June 2013.

[8] Kemal Akkaya and Mohamed Younis. A survey onrouting protocols for wireless sensor networks. Ad Hoc Networks, 3:325-349, 2005.

[9] Katayoun Sohrabi, Jay Gao, Vishal Ailawadhi, and Gregory J Pottie. Protocols for self-organization of a wireless sensor network. IEEE Personal Communications, 7:16-27, 2000.

[10] Emad Felemban, Student Member, Chang gun Lee, and Eylem Ekici. Mmspeed: Multipath multi-speed protocol for qos guarantee of reliability and timeliness in wireless sensor networks. IEEE Trans. On Mobile Computing, 5:738-754, 2006

[11] Sinem Coleri Ergen and Pravin Varaiya. Energy efficient routing with delay guarantee for sensor networks. ACM Wireless Networks, 2006.

[12] S. Ratnaraj, S. Jagannathan, and V. Rao. Oedsr:Optimized energy-delay sub-network routing in wireless sensor network. In Networking, Sensing and Control, 2006. ICNSC '06. Proceedings of the 2006 IEEE International Conference on, pages 330-335, 2006.

[13] David B. Johnson and David A. Maltz. Dynamic source routing in ad hoc wireless networks. In Mobile 
Computing, pages 153-181. Kluwer Academic Publishers, 1996.

[14] Min Chen, Victor C.M.Leung, Shiwen Mao, and Yong Yuan. Directional geographical routing for real-time video communications in wireless sensor networks. Comput. Commun., 30(17):3368-3383, November 2007.

[15] Luis Cobo, Alejandro Quintero, and Samuel Pierre. Antbased routing for wireless multimedia sensor networks using multiple qos metrics. Comput. Netw., 54(17):2991-3010, December 2010.

[16] Huang Haiping and Wang Ruchuan. Clustered control algorithm for wireless multimedia sensor network communications. In Proceedings of the 2010 International Conference on Communications and Mobile Computing- Volume 03, CMC '10, pages 264-
268, Washington, DC, USA, 2010. IEEE Computer Society.

[17] Zhen Zuo, Qin Lu, and Wusheng Luo. A two-hop clustered image transmission scheme for maximizing network lifetime in wireless multimedia sensor networks. Comput. Commun., 35(1):100-108, January 2012.

[18] Ya Xu, John Heidemann, and Deborah Estrin Geography informed energy conservation for ad hoc routing. In Proceedings of the 7 th annual international conference on Mobile computing and networking, MobiCom '01, pages 70-84, New York,NY, USA, 2001. ACM.

[19] Alberto Cerpa and Deborah Estrin. Ascent: Adap tive self-configuring sensor networks topologies,2004. 